Check for updates

Cite this: J. Mater. Chem. A, 2019, 7, 25032

\section{Highly proton conductive membranes based on carboxylated cellulose nanofibres and their performance in proton exchange membrane fuel cells $\dagger$}

\author{
Valentina Guccini, ț Annika Carlson,,$_{+}^{\mathrm{c}}$ Shun Yu, (D) $\S^{\mathrm{ab}}$ Göran Lindbergh, (D)c \\ Rakel Wreland Lindström*c and German Salazar-Alvarez (D)*ab
}

\begin{abstract}
The performance of thin carboxylated cellulose nanofiber-based (CNF) membranes as proton exchange membranes in fuel cells has been measured in situ as a function of CNF surface charge density (600 and $1550 \mu \mathrm{mol} \mathrm{g}{ }^{-1}$ ), counterion $\left(\mathrm{H}^{+}\right.$or $\left.\mathrm{Na}^{+}\right)$, membrane thickness and fuel cell relative humidity ( $\mathrm{RH} 55$ to 95\%). The structural evolution of the membranes as a function of $\mathrm{RH}$, as measured by Small Angle $\mathrm{X}$-ray Scattering, shows that water channels are formed only above $75 \% \mathrm{RH}$. The amount of absorbed water was shown to depend on the membrane surface charge and counter ions $\left(\mathrm{H}^{+}\right.$or $\left.\mathrm{Na}^{+}\right)$. The high affinity of CNF for water and the high aspect ratio of the nanofibers, together with a well-defined and homogenous membrane structure, ensures a proton conductivity exceeding $1 \mathrm{~ms} \mathrm{~cm}$ at $30{ }^{\circ} \mathrm{C}$ between 65 and $95 \% \mathrm{RH}$. This is two orders of magnitude larger than previously reported values for cellulose materials and only one order of magnitude lower than Nafion 212. Moreover, the CNF membranes are characterized by a lower hydrogen crossover than Nafion, despite being $\approx 30 \%$ thinner. Thanks to their environmental compatibility and promising fuel cell performance the CNF membranes should be considered for new generation proton exchange membrane fuel cells.
\end{abstract}

Received 10th May 2019

Accepted 17th October 2019

DOI: $10.1039 / \mathrm{c} 9 \operatorname{ta} 04898 \mathrm{~g}$

rsc.li/materials-a

\section{Introduction}

Proton exchange membrane fuel cells (PEMFC) are devices capable of producing electrical power from hydrogen gas. The high specific energy and energy density of hydrogen compared with batteries make PEMFC particularly attractive for propulsion of heavy duty and long distance vehicles. ${ }^{1} \mathrm{~A}$ critical component of the PEMFC is the membrane electrode assembly (MEA), which consists of two electrodes separated by a proton exchange membrane (PEM). The electrodes have a porous structure that allows the mass transport of the reactants and the removal of produced water. The PEMs have to meet the requirements of high proton conductivity and good electronic insulation, low fuel and oxidant permeability, low swelling and

\footnotetext{
${ }^{a}$ Department of Materials and Environmental Chemistry, Arrhenius Laboratory, Stockholm University, SE-10691 Stockholm, Sweden. E-mail: gersal@protonmail.com ${ }^{b}$ Wallenberg Wood Science Center (WWSC), Teknikringen 58, SE-100 44, Stockholm, Sweden

'Applied Electrochemistry, Department of Chemical Engineering, KTH Royal Institute of Technology, SE-100 44 Stockholm, Sweden. E-mail: rakel2@kth.se

$\dagger$ Electronic supplementary information (ESI) available: It contains details on morphological characterization, additional data on the water uptake and SAXS characterization of the membrane. File type: PDF. See DOI: 10.1039/c9ta04898g \$ Valentina Guccini and Annika Carlson contributed equally.

$\S$ Present address: RISE Research Institutes of Sweden, Lund, Sweden.
}

high stability during operation. ${ }^{2,3}$ The most commonly used membrane material is Nafion, a perfluorosulfonic acid polymer developed by Du Pont in the 1970s. Nafion membranes are characterized by high proton conductivity $\left(\approx 100 \mathrm{mS} \mathrm{cm}^{-1}\right)$ which strongly depends on the fuel cell operation parameters. ${ }^{4,5}$ Currently it is theorized that the protons are transported through water channels formed as the Nafion polymer orients into hydrophobic and hydrophilic domains. At lower water content, i.e., drier conditions, the hydrophilic domains lose continuity, and thus the ionic mobility and conductivity of Nafion membranes decrease. ${ }^{5-8}$ This has been shown by an exponential decrease when the relative humidity (RH) is lowered from 90 and $50 \% .^{9-13}$

The increased demand and interest for cost effective and environmentally benign materials other than Nafion $^{2,14,15}$ have pushed the research efforts to study alternative membranes characterized by a wider range of operation conditions in terms of RH and temperature without sacrificing the performance.

In this regard, thanks to their chemical stability, advantageous mechanical and optical properties, environmental benignity, availability and versatility in the manufactory process, ${ }^{16,17}$ the use of cellulose nanomaterials in energy applications such as lithium ion batteries ${ }^{18-22}$ and PEMFC itself ${ }^{23,24}$ has increased dramatically in recent years ${ }^{25-27}$ Several different types of nanocelluloses ${ }^{16}$ have been investigated in PEMFCs: 
bacterial cellulose (BC) membranes have shown a low conductivity $\approx 0.008 \mathrm{mS} \mathrm{cm}^{-1}$ at $40{ }^{\circ} \mathrm{C}$ and $98 \% \mathrm{RH} \cdot{ }^{28}$ Bayer et al. studied carboxylated cellulose nanofibers (CNF) and cellulose nanocrystals (CNC) membranes as PEM for high temperature application and obtained a slightly higher performance characterized by a maximum conductivity value at $100 \% \mathrm{RH}$ of 0.05 $\mathrm{mS} \mathrm{cm} \mathrm{cm}^{-1}\left(100{ }^{\circ} \mathrm{C}\right)$ and $0.01 \mathrm{mS} \mathrm{cm}^{-1}\left(30{ }^{\circ} \mathrm{C}\right)$ using an ex situ measurement replicating the fuel cell environment. ${ }^{23}$ Jankowska et $a .^{24}$ compared the performance of several nano- and microcelluloses and observed a maximum proton conductivity of $\approx 0.001 \mathrm{mS} \mathrm{cm}{ }^{-1}$ at $90{ }^{\circ} \mathrm{C}$ under non-controlled $\mathrm{RH}$ conditions. The reported values clearly show that the proton conductivity is still below that of Nafion and similar alternatives by orders of magnitude. ${ }^{15}$ The results also show that the details on the nature and dimensions of cellulose have a strong impact on the performance. Fine-tuning of the materials at the nanofiber level as well as membrane level can offer multiple ways to vastly improve their performance without compromising the many benefits of nanocellulose.

In this study, we have evaluated the in situ fuel cell conductivity of ultrathin CNF possessing different concentrations of surface carboxylate groups casted as membranes of varying thickness. The conductivity as a function of RH was correlated with the membranes water uptake and the evolution of their internal structure was determined using Small Angle Xray Scattering (SAXS). We found a proton conductivity in excess of $1 \mathrm{mS} \mathrm{cm}^{-1}$ at $30{ }^{\circ} \mathrm{C}$ and between 65 and 95\% RH, i.e., around two orders of magnitude larger than previously reported values, and relatively independent of the humidity values. The membranes were also found to be chemically stable under the operating conditions. Our results show that the CNF membranes have the potential to reach a performance close to that of traditional Nafion membranes, making it a promising and relevant candidate for PEM fuel cells.

\section{Experimental}

\section{CNF preparation}

The carboxylated CNF were prepared by TEMPO-mediated oxidation of cellulose pulp, followed by mechanical fibrillation of the oxidized pulp. The never-dried cellulose pulp was supplied by Domsjö Fabriker AB (Domsjö, Sweden). The pulp was washed with a solution of $\mathrm{HCl}$ at $\mathrm{pH} 2$, after which it was oxidized following the protocol of Saito et al. ${ }^{21,29}$ Typically, $40 \mathrm{~g}$ (dry content) of pulp was suspended in $2 \mathrm{~L}$ of deionized water and stirred together with the TEMPO catalyst $(4 \mathrm{mmol})$ and sodium bromide $(40 \mathrm{mmol})$ for $1 \mathrm{~h}$. A solution of $0.5 \mathrm{M}$ sodium hydroxide was used to adjust the $\mathrm{pH}$ to 10 and to keep it constant during the reaction, while sodium hypochlorite (9 wt\% active chlorine) was slowly added. Specifically, 37.5 and $80 \mathrm{mmol}$ of sodium hypochlorite were added to obtain, respectively, 600 and $1550 \mu \mathrm{mol} \mathrm{g}{ }^{-1}$ of carboxylic groups. After the chemical treatment, the oxidized pulp was washed with deionized water and re-dispersed with a concentration of $1 \mathrm{wt} \%$. The mechanical fibrillation was performed in two steps with a microfluidizer M-110EH, Microfluidics Corp. Firstly, the pulp was passed 3 times through two chambers with a channel size of
400 and $200 \mu \mathrm{m}$ at $925 \mathrm{bar}$, then 9 times through two smaller chambers of 200 and $100 \mu \mathrm{m}$ at 1600 bar. After the chemical treatment, part of the $1 \mathrm{wt} \%$ transparent $\mathrm{CNF}$ gel was diluted to $0.3 \mathrm{wt} \%$ and sonicated for $8 \mathrm{~min}$ with a probe sonicator $(20 \mathrm{kHz}$, $80 \%$ total power, $250 \mathrm{~mL}$ max volume). The suspension was centrifuged to remove the impurities after the sonication and the $0.3 \mathrm{wt} \% \mathrm{CNF}$ suspension was ready to use. Both sonicated and non-sonicated CNF membranes were tested in the in situ FC.

\section{CNF size and charge determination}

The length and height of CNF (see ESI Fig. S1 $\dagger$ ) were determined from the atomic force micrographs (Veeco Dimension 3100 SPM) obtained using tapping mode in air. The samples were prepared by depositing $300 \mu \mathrm{L}$ of $0.01 \mathrm{wt} \% \mathrm{CNF}$ suspension onto mica substrates pre-treated with 3-aminopropyl triethoxysilane (Sigma Aldrich, 99\%). The excess of suspension was removed by a stream of air. The surface charge density was determined by conductometric titration on the oxidized pulp following the protocol reported in literature. ${ }^{21}$

\section{Membrane casting and characterization}

The CNF membranes were casted in Petri dishes with a diameter of $5.5 \mathrm{~cm}$. The appropriate amount of $0.3 \mathrm{wt} \% \mathrm{CNF}$ suspension was dried at $30{ }^{\circ} \mathrm{C}$ and $50 \% \mathrm{RH}$ for 3 days. The thickness of the CNF membranes was measured by a highaccuracy digital micrometer MDH-25MB (Mitutoyo) with a precision of $0.1 \mu \mathrm{m}$. The images of the surface and the crosssection of the membrane were obtained by scanning electrode microscope (SEM) JEOL JSM-7401F.

\section{Membrane water uptake and ion exchange}

The cellulose membranes were ion-exchanged by submerging them in solution of $0.01 \mathrm{M}$ sulfuric acid for $30 \mathrm{~min}$ and then rinsed in Milli-Q water until neutral $\mathrm{pH}$. The membranes before the ion exchange are named CNF-COONa, while the ones after the ion exchange are CNF-COOH. The Nafion 212 membrane was used as received. The CNF membranes water uptake was measured at $30{ }^{\circ} \mathrm{C}$ and $55,65,75,85$ and $95 \%$ RH by conditioning the membrane for two days in controlled humidity and temperature condition. The increase of weight due to the water absorption was measured with a balance with a $10 \mu \mathrm{g}$ precision. Each measurement corresponds to the average of three different replicas. The water uptake was calculated according to the following equation

$$
W \%(\mathrm{RH})=\left(W_{\mathrm{RH}}-W_{\text {dry }} / W_{\text {dry }}\right) \times 100
$$

in which $W_{\mathrm{RH}}$ is the weight of the membrane at specific $\mathrm{RH}$ condition and $W_{\text {dry }}$ is the weight of the dry membrane, dried at $105{ }^{\circ} \mathrm{C}$ overnight before the measurement.

\section{In situ FC electrochemical characterization}

After the ion exchange the membranes were mounted in a Fuel Cell Technologies cell housing between two $2.5 \mathrm{~cm}^{2}$ commercial $0.5 \mathrm{mg} \mathrm{Pt} \mathrm{cm}^{-2}$ on cloth gas diffusion electrodes (Fuel Cells Etc). 
The cell was transferred to a fuel cell set-up and heated to a cell temperature of $30{ }^{\circ} \mathrm{C}$ and $95 \% \mathrm{RH}$ under nitrogen flow and left to equilibrate for $2 \mathrm{~h}$ in order to ensure similar initial conditions for all tests. The experiments were performed as follows: impedance (open-circuit, amplitude $1 \mathrm{~mA}, 100 \mathrm{kHz}$ to $1 \mathrm{~Hz}$, under $\mathrm{N}_{2} / \mathrm{N}_{2}$ gas), cyclic voltammetry (30 $\mathrm{mV} \mathrm{s}^{-1}, 0.1-1.2 \mathrm{~V}$, under $\mathrm{N}_{2} / \mathrm{H}_{2}$ gas) and crossover current density measurements $\left(1 \mathrm{mV} \mathrm{s}^{-1}\right.$, OCP-0.7 $\mathrm{V}$, under $\mathrm{N}_{2} / \mathrm{H}_{2}$ gas) were performed using 60 $\mathrm{mL} \min ^{-1}$ nitrogen flow and $30 \mathrm{~mL} \mathrm{~min}^{-1}$ hydrogen flow. Thereafter, current-voltage curves were measured from Open Circuit Voltage (OCV) to $0.3 \mathrm{~V}$ at a scan rate of $1 \mathrm{mV} \mathrm{s}^{-1}$ with 50

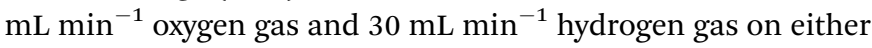
side of the membrane. The change in conductivity and gas crossover with relative humidity was measured after the sequence above going from high humidity to low with nitrogen gas at the working electrode and hydrogen gas at the counter electrode.

\section{Small angle X-ray scattering}

The measurements were carried out at the beamline P03 "MiNaXS", of PETRA III storage ring, at the Deutsches Elektronen-Synchrotron (DESY), Germany. The incident X-ray beam had a wavelength of $0.0957 \mathrm{~nm}$ and a beam size of about $20 \times 20 \mu^{2}$. The sample-to-detector distance was calibrated by dry rat-tail collagen to $2500 \pm 0.1 \mathrm{~mm}$. The scattering patterns were recorded using a $2 \mathrm{D}$ pixel detector (Pilatus $1 \mathrm{M}$ ). To minimize beam damage and obtain representative measurements, the samples were scanned at seven different sample positions and their 1D scattering pattern $(I(q))$ was extracted via radial integration as a function of the wavevector $q$. The $I(q)$ were normalized by the Porod invariant $Q$ to compensate for the change in scattering intensity, $I$, due to the variation of CNF volume fraction, $\varphi$ and to highlight the structural information. $\int_{0}^{\infty} q^{2} I(q) \mathrm{d} q=2 \pi^{2} \Delta \rho^{2} \varphi(1-\varphi)$ where $\Delta \rho$ is the electron density difference between CNF and water. Stripes of the CNF membranes (thickness $\approx 15 \mu \mathrm{m}$ ) were mounted in a conditioned chamber with control humidity and temperature. Each sample was conditioned for 2 days at each specific humidity (55, 65, 75, 85 and 95\%) and sealed. Prior to the measurement, the membrane was conditioned inside the chamber for additional $15 \mathrm{~min}$. The acquisition time for each measurement was $0.5 \mathrm{~s}$ for the membrane conditioned at 95 and $85 \% \mathrm{RH}$ and $1 \mathrm{~s}$ for the other conditions. The acquisition times were selected after comparing the intensity and the scattering profile changes under continuous X-ray beam exposure.

\section{Results}

The CNF membranes were manufactured from an aqueous suspension of nanofibers characterized by high aspect ratio with a length of $\approx 300 \mathrm{~nm}$ and height of $\approx 2 \mathrm{~nm}$ (see ESI Fig. $\mathrm{S} 1 \dagger$ ). Moreover, the CNF are characterized by high stability in acid conditions up to $30{ }^{\circ} \mathrm{C}$ (see ESI $\dagger$ ). This was also studied in detail by Fujisawa et al. on celluronic acid obtained by TEMPO mediated oxidized cellulose at $\mathrm{pH} 10 .^{30}$ The water uptake and conductivity as a function of relative humidity of CNF membranes consisting of nanofibers with a surface charge density of 600 and $1550 \mu \mathrm{mol} \mathrm{g}{ }^{-1}$ (CNF-600 and CNF-1550, respectively) is shown in Fig. 1. Fig. 1A shows the water uptake of the CNF-600 and CNF-1550 membranes before and after ion exchange (see also Table S1 ESI $\dagger$ ).

The water uptake for the $\mathrm{CNF}-\mathrm{COOH}$ increases about one order of magnitude from 55 to $95 \% \mathrm{RH}$, while for the CNFCOONa ones increases two orders of magnitude. At 95\% RH the water uptake of $\mathrm{CNF}-\mathrm{COONa}$ is approximately $5 \times$ larger than that of $\mathrm{CNF}-\mathrm{COOH}$. The CNF membranes with higher surface charge density $\left(1550 \mu \mathrm{mol} \mathrm{g}^{-1}\right)$ have higher water uptake compared to the ones with lower $\left(600 \mu \mathrm{mol} \mathrm{g}{ }^{-1}\right)$, in agreement with earlier reports. ${ }^{1729}$ The carboxylic groups have a lower hydration shell than the carboxylated groups thus reducing the water uptake which further translates in lower swelling and higher dimensional stability. Moreover, in $\mathrm{CNF}-\mathrm{COOH}$, due to the hydrogen bond and absence of electrostatic repulsions, the attractive interactions between cellulose nanofibers is stronger, which results in a higher Young's modulus. ${ }^{31}$

The in situ conductivity of a number of samples was measured as a function of $\mathrm{RH}$, from 95 to $55 \%$ and the results are shown in Fig. 1B. Note that the discrepancy in absolute values for the proton conductivity in Nafion measured in this work and the values in the literature is likely related to MEA preparation and internal resistances within the cell housing.

At $95 \% \mathrm{RH}$, the conductivity of $\mathrm{H}-\mathrm{CNF}-1550$ is $\approx 2 \mathrm{mS} \mathrm{cm}^{-1}$, two orders of magnitude larger than the highest values previously reported for nanocellulose-based membranes. ${ }^{23}$ However, the conductivity of H-CNF-1550 membranes is still 10-50× lower than that of Nafion 212, both in control experiments and literature data, respectively. ${ }^{10}$ The conductivity of H-CNF-1550 is constant above $65 \%$, but drops significantly at $55 \% \mathrm{RH}$, whereas the conductivity of Nafion decreases exponentially with decreasing RH. The results shown in Fig. 1B indicate that the in situ conductivity of CNF membranes is far less sensitive to humidity changes than Nafion, probably due to the higher hydrophilic character of CNF compared to Nafion. For this reason, CNF membranes can absorb much more water compared to Nafion and thus the CNF membrane may have a conductivity less sensitive to lowered relative humidity. Namely, the water content $\lambda,{ }^{32,33}$ represented by the number of water molecules per proton conductive site in both CNF 600 and 1550 (Fig. $\mathrm{S} 2 \dagger$ ), is $>100 \times$ higher than that of Nafion $212 .^{10}$

The proton conductivity of the CNF membranes was also measured for membranes with different thicknesses, $t$, and surface charge densities.

Table 1 summarizes the conductivity values, $\sigma$, obtained by electrochemical impedance spectroscopy (EIS) in the fuel cell for H-CNF-1550 and H-CNF-600. The EIS spectra are plotted in Fig. $\mathrm{S} 8 \dagger$

The proton conductivity of the H-CNF-1550 with $t \approx 14 \mu \mathrm{m}$ is the highest amongst all the CNF membranes and is characterized by a value only one order of magnitude lower than Nafion $212\left(\approx 20 \mathrm{mS} \mathrm{cm}{ }^{-1}\right)$ when measured under the same conditions. The conductivity is observed to be independent of the membranes thickness, indicating that the membranes have 

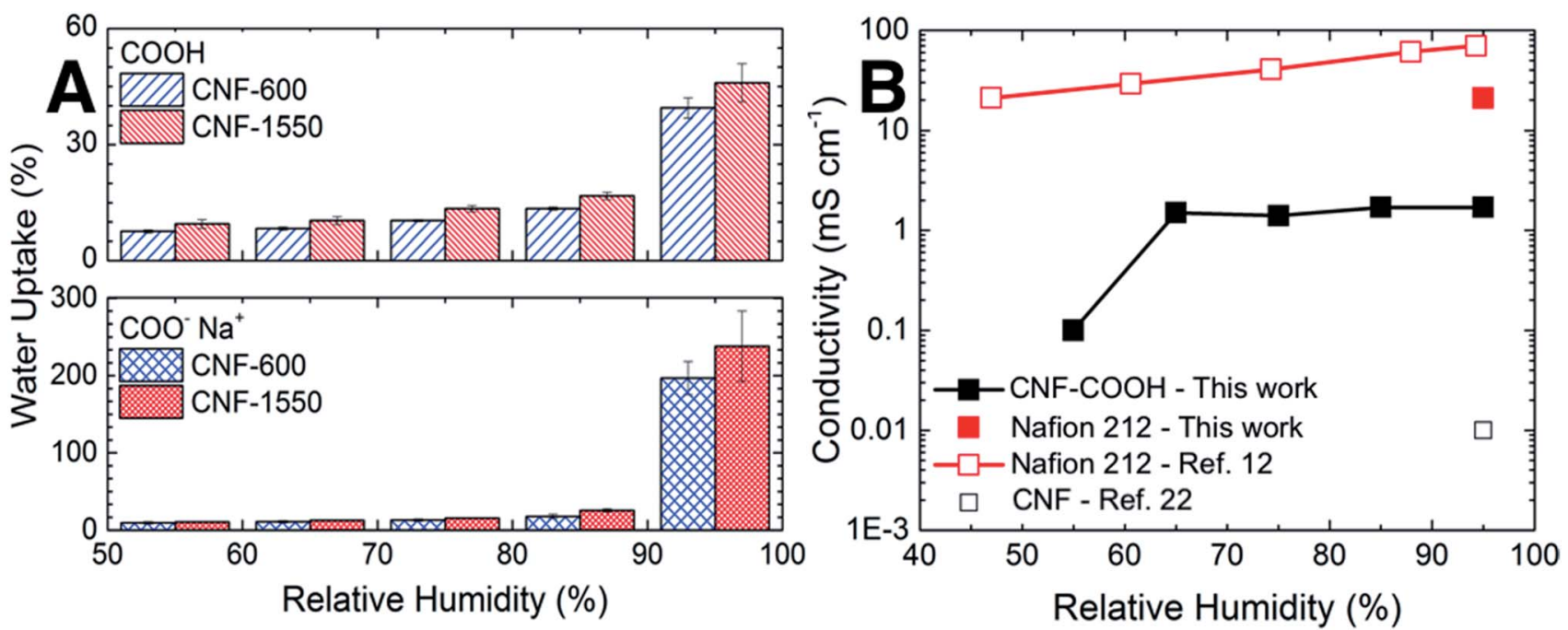

Fig. 1 (A) Water uptake of carboxylated (COONa) and carboxylic (COOH) CNF membranes. (B) Experimental conductivity of the H-CNF-1550 membrane and Nafion determined in this work (full symbols) and values from the literature (empty symbols) for Nafion 212 (ref. 12 ) and CNF. ${ }^{22}$

Table 1 Proton conductivity, $\sigma$, of $\mathrm{H}-\mathrm{CNF}$ membranes as a function of thickness, $t$, measured under $\mathrm{N}_{2} / \mathrm{N}_{2}$ gas, at $30^{\circ} \mathrm{C}$ and $95 \% \mathrm{RH}$. Standard deviation calculated from 2 membranes

\begin{tabular}{lll}
\hline & \multicolumn{2}{l}{ Proton conductivity, $\sigma\left(\mathrm{mS} \mathrm{cm}^{-1}\right)$} \\
\cline { 2 - 3 } Sample & $t \approx 14 \mu \mathrm{m}$ & $t \approx 24 \mu \mathrm{m}$ \\
\hline H-CNF-600 & $1.4 \pm 0.1$ & $1.2 \pm 0.4$ \\
H-CNF-1550 & $1.5 \pm 0.2$ & $1.4 \pm 0.2$
\end{tabular}

a homogenous internal structure. The large conductivity observed in Nafion is ascribed to the large amount and the high acidity of the sulfonic group $\left(\mathrm{p} K_{\mathrm{a}} \approx-6\right)^{34}$ as well as the presence of confined water channels between its hydrophobic and hydrophilic domains. ${ }^{11}$ Herein, the difference between CNF and Nafion is likely due to the use of functional groups that are significantly less acidic, i.e., the $\mathrm{p} K_{\mathrm{a}}$ of carboxylic acid is $\approx 3-4 .^{35}$ Regarding the presence of water channels in CNF membranes and overall their internal structure, we characterized the membranes with scanning electron microscopy (SEM) and small angle X-ray scattering (SAXS). Fig. 2A and B show SEM images of the surface and the cross-section of the H-CNF-1500 membrane, respectively. As can be seen from the images, at the surface, the nanofibers form an entangled network ${ }^{36}$ whereas the image of the cross-section shows that the CNF are arranged together in layers. This layered structure is characteristic of CNF films dried by slow evaporation of the solvent ${ }^{37,38}$ and gives the membranes barrier properties against gases such as oxygen and hydrogen. ${ }^{39}$ Thanks to the controlled slow drying, the dried CNF films are dense and transparent.

Fig. 2C and D show the evolution of CNF membrane structure upon water absorption at increasing $\mathrm{RH}$ as studied by SAXS. The SAXS data were fitted using a two-stage model

$$
I(q)=\frac{A}{q^{n}}+B \exp \left(-\frac{q^{2} \Xi^{2}}{2}\right)+C
$$

where $A$ and $B$ are scaling factors and $C$ is a constant background. The first term to the right is a power law with a scaling exponent $n$, known as Porod exponent, which describes the complexity of the network. As RH increases $n$ decreases suggesting that, in the tested RH condition, the CNF agglomerates are kept highly entangled, despite water causing swelling of the nanofiber network. Generally, an exponent $1<n<3$ indicates that the network may adopt a bulk fractal dimension, whereas 2 $<n<3$ is known as a mass fractal dimension, which is observed in most cases in this study (see ESI Table S2 $\dagger$ ). The second term is a Gaussian function used to describe the average pore size $\Xi$ in the densely packed CNF membranes. $\Xi$ accounts for the distinct feature that appears around $q \approx 0.59 \mathrm{~nm}^{-1}$, that increases in intensity with the water content and is attributed to the formation of water channels in the pores. Indeed, due to the swelling induced by the water uptake, the size of the pores increases from $\approx 1.0 \mathrm{~nm}$ at $55-75 \%$ to $\approx 2 \mathrm{~nm}$ at $95 \% \mathrm{RH}$ (see ESI Table S2 $\uparrow$ ), i.e., the pores are still smaller than the average diameter of CNF even at these values of water uptake (Fig. 1A). At $95 \%$ RH the CNF membranes have a pore size comparable with fully hydrated Nafion with a width of $2.5 \pm 0.2 \mathrm{~nm}$, as determined with electron microscope cryotomography. ${ }^{40}$ An increase of porosity during the swelling increases the scattering volume (CNF-pore) although the incorporation of water in the membrane channels reduces the scattering contrast (CNFwater interphase), which leads to an overall increase in intensity at the high $q$ range. The higher scattering intensity of H-CNF1500 (Fig. 2C) is associated with a larger water uptake compared to H-CNF-600 (Fig. 2D), as the amount of water depends on the type of counter ion $\left(\mathrm{H}^{+}\right.$or $\left.\mathrm{Na}^{+}\right)$and on the amount of surface charge on the nanofibers (Fig. 1A). The SAXS pattern of the Na-CNF-1550 and Na-CNF-600 (SESI Fig. S3 and Table S2 $\dagger$ ) shows higher intensity and a less defined feature at high $q$, due to the larger amount of water and larger swelling. The structural evolution of the membrane as a function of humidity suggests that the formation of the water channels in 

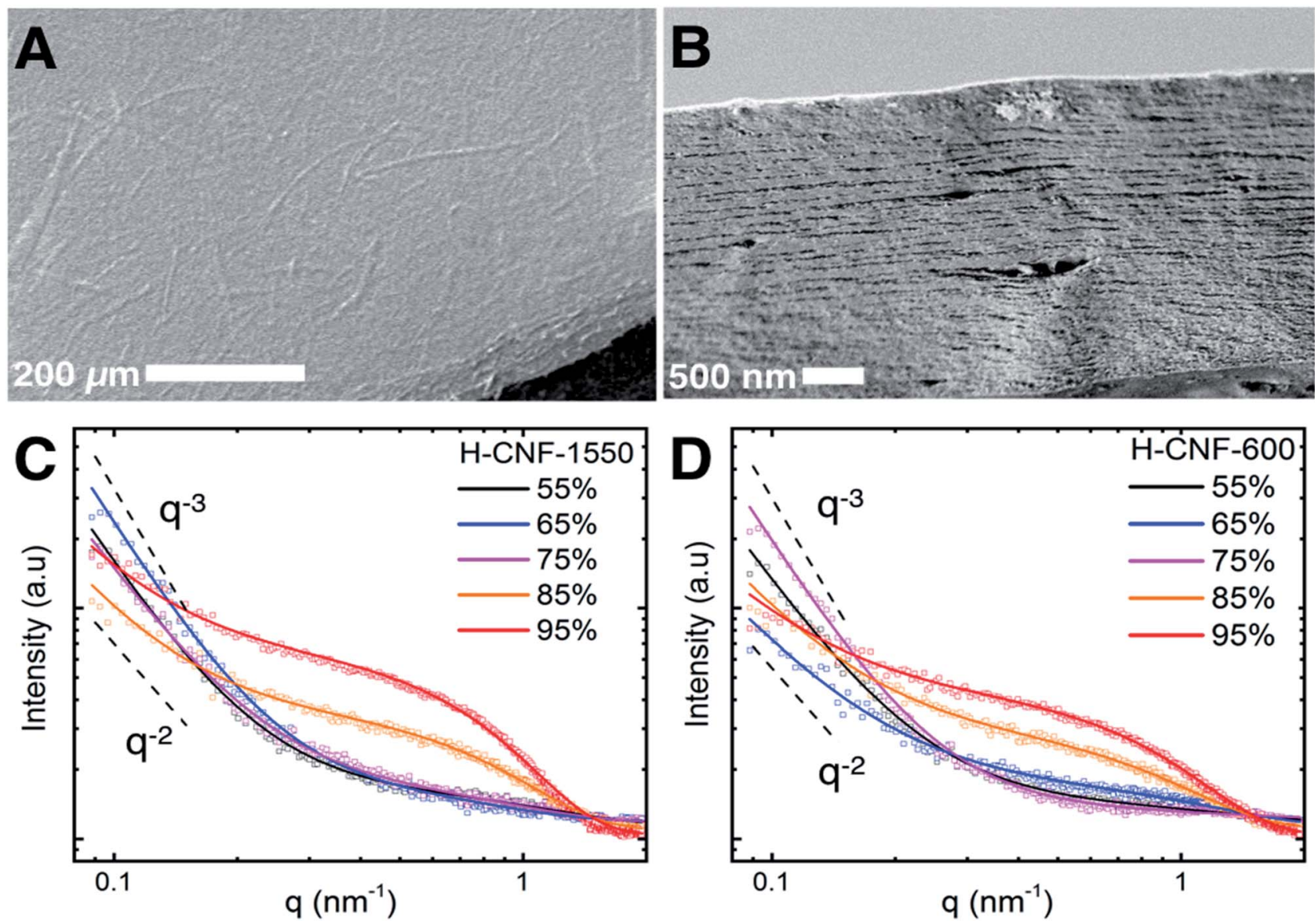

Fig. 2 SEM image of (A) the surface of a H-CNF-1550 membrane and (B) its cross-section. Radial integrated intensity SAXS profiles of (C) H-CNF1550 and (D) H-CNF-600 as a function of relative humidity (55-95\%). The lines are fits to the data using eqn (2). The slope lines are guides to the eye.

the structure happens at humidity values $>75 \%$. We speculate that the already high water content ensures a good proton conductivity and that additional water does not have an important impact in the performance as opposite to what is observed for Nafion. ${ }^{11}$ At the same time, the in situ conductivity remains constant down towards $65 \%$ RH (Fig. 1B) despite the variation in water content (Fig. 1A), meaning that bound water has to contribute to the proton conduction. The interaction between water and cellulose has been extensively studied, ${ }^{41-43}$ showing that the water is present in the CNF both as absorbed and bound water. The movable bound water is located between the nanofiber agglomerate-agglomerate interface whereas the immobile portion is between nanofibers belonging to the same agglomerate..$^{43}$ The absorbed water promotes the formation of channels with increased humidity (feature at high $q$ ). Intuitively, the mobility of the absorbed water is higher than the bound water, suggesting that the predominant and most favorable proton conduction mechanism in CNF membrane may occur via the hydrogen bonds present in the liquid water (Grötthuss mechanism). The fact that the in situ proton conductivity is constant as a function of $\mathrm{RH}(>65 \% \mathrm{RH})$ is unprecedented for nanocellulose based membranes. This phenomenon is also opposite for Nafion in which the RH plays a very large role in the membrane conductivity. ${ }^{11}$ Bayer et al. show that ex situ proton conductivity in their CNF decreases approximately 2 orders of magnitude between 80 and $70 \%$ RH. ${ }^{23}$ The lower conductivity towards low RH could be attributed to the use of CNF with fiber thicknesses of tens to hundreds of nanometers with a lower amount of surface water, $c f$. the CNF used in this study with a thickness of $\approx 2 \mathrm{~nm}$ and a high amount of surface water (ESI Fig. S1 †). Recently, Jankowska et al. showed that films made of nanocellulose had higher proton conductivity compared to the ones made of larger microcellulose ${ }^{24}$ supporting our results that highlight the importance of high aspect ratio nanofibers and well defined and homogenous membrane structures (achievable by controlled slow drying conditions). After the fuel cell evaluation, the physicochemical characterization (including SAXS, XRD and IR) show no significant alteration in their chemistry, crystallinity or microstructure (see ESI, Fig. S4 and S5†).

In addition to high proton conductivity, barrier properties towards $\mathrm{H}_{2}$ and $\mathrm{O}_{2}$ is another key factor for a well-functioning PEM, which has been previously shown for CNF. ${ }^{39}$

Here, the in situ characterization of the $\mathrm{H}_{2}$ permeability, was performed using cyclic voltammetry (CV) and crossover current density measurements. Fig. 3A shows the cyclic voltammograms 

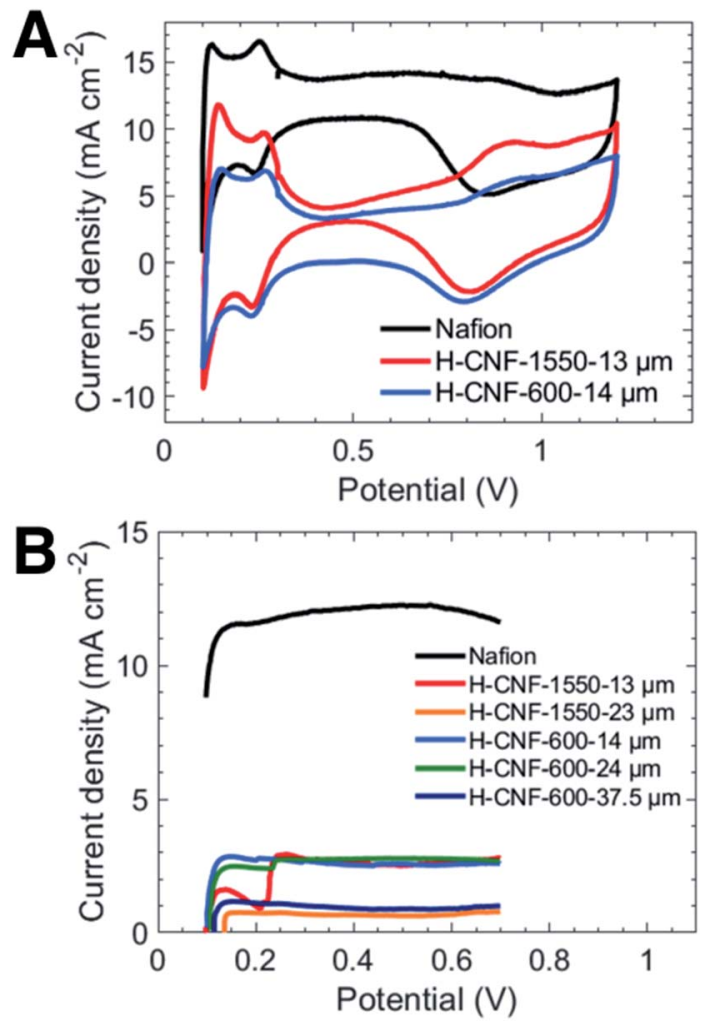

Fig. 3 Hydrogen crossover characterization of $\mathrm{H}$-CNF membranes compared to Nafion 212. (A) Cyclic voltamogram of the H-CNF membrane as a function of surface charge density. (B) Hydrogen crossover current density of the H-CNF membranes as a function of surface charge density and thickness. Measurements obtained at $95 \%$ $\mathrm{RH}$ and $30^{\circ} \mathrm{C}$.

of the H-CNF membranes with 600 and $1550 \mu \mathrm{mol} \mathrm{g}{ }^{-1}$ and Nafion 212. Both CNF and Nafion curves show similar size of the adsorption and desorption peaks for hydrogen onto the 100 and 110 facets of the Pt catalyst (peaks below $0.4 \mathrm{~V}$ ). This indicates that the electrodes in all tests have similar amounts of catalytic sites available and that the use of CNF membranes does not affect the catalyst layer. At $\mathrm{N}_{2} / 100 \% \mathrm{H}_{2}$ conditions in a fuel cell the crossover of hydrogen from the counter/reference electrode results in a typical lift of the $\mathrm{CV}$ for Nafion. However, both H-CNF membranes curves center around a lower current density than Nafion, which is an indication of a lower hydrogen crossover at these operating conditions, despite the CNF membranes being are significantly thinner ( $\approx 14 \mu \mathrm{m}$ compared to $51 \mu \mathrm{m})$. The low hydrogen permeability is probably related to the layered structure and high density of the CNF membranes (Fig. 2A and B). The same conclusions were reached for CNF and CNC membrane prepared by vacuum filtration and hot pressing. ${ }^{23}$ The crossover current density (Fig. 3B), proportional to the hydrogen crossover, confirms the observations in the CV characterization. Nafion is characterized by the highest crossover current density and among the CNF membranes the H-CNF-600 has the lowest crossover. No significant difference between $\mathrm{H}$ CNF-600 and H-CNF-1550 is observed, but a small decrease can be seen with increased membrane thickness. Calculating the permeability coefficients for the CNF membranes gives $1.5 \pm 0.5$ $\times 10^{-11}$ and $2 \pm 0.5 \times 10^{-11} \mathrm{~mol} \mathrm{~cm}^{-1} \mathrm{~s}^{-1}$ bar $^{-1}$ for the thicker and thinner membranes respectively. The values are very close and indicate that the permeability does not change by altering the thickness. For Nafion the corresponding value in our setup and cell housing is an order of magnitude higher, i.e., $3.1 \times$ $10^{-10} \mathrm{~mol} \mathrm{~cm}^{-1} \mathrm{~s}^{-1} \mathrm{bar}^{-1}$. With increased $\mathrm{RH}$, the hydrogen crossover of Nafion membranes also increases as the greater distance between polymer chains (due to the water channels) offers additional pathways for the crossover. ${ }^{44}$ In the case of the CNF membrane the opposite behavior is observed. Indeed, the Nyquist plots (Fig. S9†) show that the crossover is higher at lower $\mathrm{RH}$. Most probably when the absorbed water forms channels (shown by SAXS) at high humidity, once the water content inside the membrane is lowered, these channels give the additional pathways for the diffusion of gas. This hypothesis is strengthened when assessing the durability of the CNF membranes (Fig. S10 $\dagger$ ). Indeed, after the RH of the cell was decreased from $95 \%$ to $85 \%$ and brought back to $95 \%$, the OCV is lower than the initial value, indicating a larger cross-over.

The fuel cell performance of the investigated membranes is shown in Fig. 4.

The influence of the CNF surface charge density and thickness of membranes on performance was evaluated by current density-voltage measurements in a fuel cell during operation at $30{ }^{\circ} \mathrm{C}$ and $95 \% \mathrm{RH}$. The IR-corrected (dashed lines) and non-
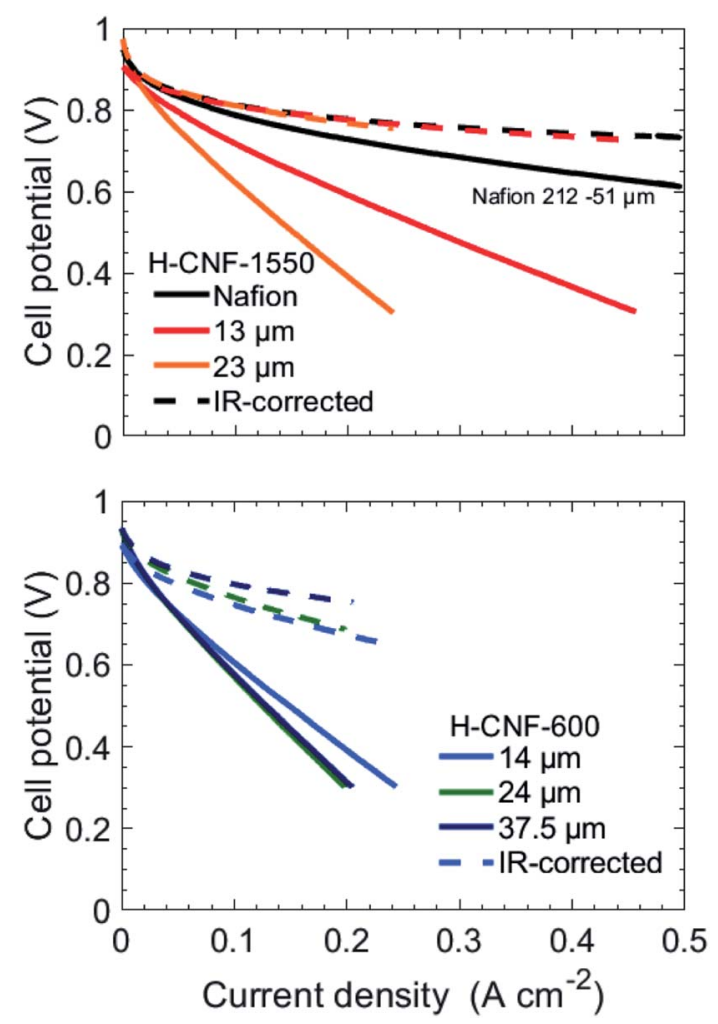

Fig. 4 Polarization curves of CNF membranes as a function of surface charge density and thickness. Caption polarization, as well Nafion 212 $\left(95 \% \mathrm{RH}\right.$ and $30{ }^{\circ} \mathrm{C}$ ). The dashed curves represent IR-corrected measurements. 
corrected (solid lines) polarization curves of $\mathrm{H}-\mathrm{CNF}-1550$ and $\mathrm{H}-$ CNF-600 membranes at different thicknesses are shown. The $I R$ corrected lines are compensated using the high frequency resistance, from which the conductivity was calculated, and removes any differences in resistance due to various thickness of the membranes and any other resistive losses in the cell.

All the CNF membranes have a high open circuit voltage, close to that of Nafion, which is expected from the low hydrogen crossover. In the current voltage measurements, the H-CNF1550 membranes show a superior performance to H-CNF-600, although still having a higher resistance than Nafion. These results are in accordance with the conductivity measurements above. The higher performance of the H-CNF-1550 membranes compared to the H-CNF-600 is the result of slightly smaller dimensions, which leads to a higher water uptake and a more pronounced formation of water channels, as observed by SAXS measurements. During the durability measurement a performance loss was observed at $85 \% \mathrm{RH}$ for the H-CNF-1550 membrane, but after increasing the RH to $95 \%$ the cell performance and conductivity were partially recovered and stable throughout the experiment (Fig. S10 $\dagger$ ). As discussed, the lower conductivity and so, lower performance compared to Nafion is likely related to the lower acidity of the carboxylic groups but the interfacial resistance between electrode and membrane, and/or differences in membrane structure may play a role. However, the good crossover properties of very thin CNF membranes show promise to reduce the internal resistance of the fuel cell. Further, as the CNF films are characterized by better mechanical properties than Nafion, they could provide a robust alternative in several energy applications. For example Nafion 117 at $30 \% \mathrm{RH}$ have Young's modulus of $\approx 0.2 \mathrm{GPa},{ }^{45}$ whereas Na-CNF films show $\approx 15 \mathrm{GPa} .{ }^{31}$ However, for both CNF and Nafion films the Young's modulus decreases with an increased relative humidity. These CNF membranes are very promising for PEMFC applications, but further optimization of the MEA under varying fuel cell operating conditions is required.

\section{Conclusions}

The surface chemistry and small dimensions of CNF used in this work have a profound influence in their performance as proton conducting membranes. The membranes presented in this work show proton conductivity values $10-50 \times$ lower than Nafion. However, the membranes outperform Nafion in terms of gas barrier properties and much lower sensitivity to changes in RH. Indeed, the number of water molecules per proton conductive site is more than 100 times higher than that for Nafion 212. Our results suggest that the bound water CNF has a key role in ensuring the proton conduction independently to the amount of bulk absorbed water and gives a dominant contribution specially at low RH. Further, thanks to the superior mechanical properties of CNF membranes compared to Nafion, they can be fabricated to be thinner, potentially reducing the internal cell resistance, without compromising the gas barrier properties. Lastly, our study shows that CNF membranes with their environmental compatibility, renewable origin, low gas crossover and promising fuel cell performance should be considered for use in a new generation of PEMFCs.

\section{Conflicts of interest}

There are no conflicts to declare.

\section{Acknowledgements}

The authors thank Sugam Kumar, Luis Valencia, Aji P. Mathew and Peng Liu for the experimental support during the beam time and Zoltán Bacsik for scientific discussions. The Knut and Alice Wallenberg foundation is acknowledged for the financial support through the Wallenberg Wood Science Center. The research was also partially sponsored by the Swedish Vehicle Research and Innovation program, the Swedish Energy Agency and the Swedish governmental initiative StandUp for Energy. SY thanks the financial support from the Swedish Research Council VR (Grant 2016-06959). We acknowledge DESY (Hamburg, Germany), a member of the Helmholtz Association HGF, for the provision of experimental facilities. Parts of this research were carried out at PETRA III and we would like to thank Wiebke Ohm for assistance in using the beamline P03. This work benefited from the use of the SasView application, originally developed under NSF award DMR-0520547. SasView contains code developed with funding from the European Union's Horizon 2020 research and innovation programme under the SINE2020 project, grant agreement no. 654000.

\section{Notes and references}

1 Z. P. Cano, D. Banham, S. Ye, A. Hintennach, J. Lu, M. Fowler and Z. Chen, Nat. Energy, 2018, 3, 279-289.

2 B. Steel and A. Heinzel, Nature, 2001, 414, 345-352.

3 Y. Wang, K. S. Chen, J. Mishler, S. C. Cho and X. C. Adroher, Appl. Energy, 2011, 88, 981-1007.

4 B. Smitha, S. Sridhar and A. A. Khan, J. Membr. Sci., 2005, 259, 10-26.

5 K. A. Mauritz and R. B. Moore, Chem. Rev., 2004, 104, 45354586.

6 O. Savadogo, J. Power Sources, 2004, 127, 135-161.

7 S. Bose, T. Kuila, T. X. H. Nguyen, N. H. Kim, K. T. Lau and J. H. Lee, Prog. Polym. Sci., 2011, 36, 813-843.

8 R. E. Rosli, A. B. Sulong, W. R. W. Daud, M. A. Zulkifley, T. Husaini, M. I. Rosli, E. H. Majlan and M. A. Haque, Int. J. Hydrogen Energy, 2017, 42, 9293-9314.

9 M. Schalenbach, W. Lueke, W. Lehnert and D. Stolten, Electrochim. Acta, 2016, 214, 362-369.

10 Q. He, A. Kusoglu, I. T. Lucas, K. Clark, A. Z. Weber and R. Kostecki, J. Phys. Chem. B, 2011, 115, 11650-11657.

11 M. L. Einsla, Y. S. Kim, M. Hawley, H. S. Lee, J. E. McGrath, B. Liu, M. D. Guiver and B. S. Pivovar, Chem. Mater., 2008, 20, 5636-5642.

12 A. V. Anantaraman and C. L. Gardner, J. Electroanal. Chem., 1996, 414, 115-120.

13 J. Zhang, Y. Tang, C. Song, Z. Xia, H. Li, H. Wang and J. Zhang, Electrochim. Acta, 2008, 53, 5315-5321. 
14 R. S. L. Yee, R. A. Rozendal, K. Zhang and B. P. Ladewig, Chem. Eng. Res. Des., 2012, 90, 950-959.

15 K.-D. Kreuer, Chem. Mater., 2014, 26, 361-380.

16 D. Klemm, F. Kramer, S. Moritz, T. Lindström, M. Ankerfors, D. Gray and A. Dorris, Angew. Chem., Int. Ed. Engl., 2011, 50, 5438-5466.

17 R. J. Moon, A. Martini, J. Nairn, J. Simonsen and J. Youngblood, Chem. Soc. Rev., 2011, 40, 3941-3994.

18 S. Leijonmarck, A. Cornell, G. Lindbergh and L. Wågberg, Nano Energy, 2013, 2, 794-800.

19 S. Leijonmarck, A. Cornell, G. Lindbergh and L. Wågberg, J. Mater. Chem. A, 2013, 1, 4671.

$20 \mathrm{H}$. Lu, M. Behm, S. Leijonmarck, G. Lindbergh and A. Cornell, ACS Appl. Mater. Interfaces, 2016, 8, 18097-18106.

21 H. Lu, V. Guccini, H. Kim, G. Salazar-Alvarez, G. Lindbergh and A. Cornell, ACS Appl. Mater. Interfaces, 2017, 9, 3771237720 .

22 H. Kim, V. Guccini, H. Lu, G. Salazar-Alvarez, G. Lindbergh and A. Cornell, ACS Appl. Energy Mater., 2019, 2, 1241-1250.

23 T. Bayer, B. V. Cunning, R. Selyanchyn, M. Nishihara, S. Fujikawa, K. Sasaki and S. M. Lyth, Chem. Mater., 2016, 28, 4805-4814.

24 I. Jankowska, R. Pankiewicz, K. Pogorzelec-Glaser, P. Ławniczak, A. Łapiński and J. Tritt-Goc, Carbohydr. Polym., 2018, 200, 536-542.

25 B. Wicklein and G. Salazar-Alvarez, J. Mater. Chem. A, 2013, 1, 5469.

26 N. Muhd Julkapli and S. Bagheri, Polym. Adv. Technol., 2017, 28, 1583-1594.

27 W. Chen, H. Yu, S. Y. Lee, T. Wei, J. Li and Z. Fan, Chem. Soc. Rev., 2018, 47, 2837-2872.

28 T. D. O. Gadim, F. J. A. Loureiro, C. Vilela, N. Rosero-Navarro, A. J. D. Silvestre, C. S. R. Freire and F. M. L. Figueiredo, Electrochim. Acta, 2017, 233, 52-61.

29 A. Isogai, T. Saito and H. Fukuzumi, Nanoscale, 2011, 3, 7185.
$30 \mathrm{~S}$. Fujisawa, T. Isogai and A. Isogai, Cellulose, 2010, 17, 607615.

31 A. Benítez, J. Torres-Rendon, M. Poutanen and A. Walther, Biomacromolecules, 2013, 14, 4497-4506.

32 T. A. Zawodzinski, S. Gottesfeld, S. Shoichet and T. J. McCarthy, J. Appl. Electrochem., 1993, 23, 86-88.

33 T. A. Zawodzinski, J. Davey, J. Valerio and S. Gottesfeld, Electrochim. Acta, 1995, 40, 297-302.

34 K. D. Kreuer, J. Membr. Sci., 2001, 185, 29-39.

35 Scandinavian Pulp, Paper and Board Testing Committee, SCAN Test method CM 65:02, 2002, vol. 1-4.

36 V. Guccini, S. Yu, M. Agthe, K. Gordeyeva, Y. Trushkina, A. Fall, C. Schütz and G. Salazar-Alvarez, Nanoscale, 2018, 10, 23157-23163.

37 M. Nogi, S. Iwamoto, A. N. Nakagaito and H. Yano, Adv. Mater., 2009, 21, 1595-1598.

38 M. Zhao, F. Ansari, M. Takeuchi, M. Shimizu, T. Saito, L. A. Berglund and A. Isogai, Nanoscale Horiz., 2018, 3, 28-34.

39 H. Fukuzumi, T. Saito, S. Iwamoto, Y. Kumamoto, T. Ohdaira, R. Suzuki and A. Isogai, Biomacromolecules, 2011, 12, 4057-4062.

40 F. I. Allen, L. R. Comolli, A. Kusoglu, M. A. Modestino, A. M. Minor and A. Z. Weber, ACS Macro Lett., 2015, 4, 1-5. 41 J. Berthold, J. Desbrières, M. Rinaudo and L. Salmén, Polymer, 1994, 35, 5729-5736.

42 H. O'Neill, S. V. Pingali, L. Petridis, J. He, E. Mamontov, L. Hong, V. Urban, B. Evans, P. Langan, J. C. Smith and B. H. Davison, Sci. Rep., 2017, 7, 11840.

43 E. L. Lindh, C. Terenzi, L. Salmén and I. Furó, Phys. Chem. Chem. Phys., 2017, 19, 4360-4369.

44 K. D. Baik, B. K. Hong and M. S. Kim, Renewable Energy, 2013, 57, 234-239.

45 Y. Tang, A. M. Karlsson, M. H. Santare, M. Gilbert, S. Cleghorn and W. B. Johnson, Mater. Sci. Eng., A, 2006, 425, 297-304. 